\title{
New Insights into Annual and Semiannual Cycles of Sea Level Pressure
}

\author{
Ge CHEN AND CHENGCHENG QIAN \\ Department of Marine Technology, College of Information Science and Engineering, Ocean University \\ of China, Qingdao, China \\ CAIYUN ZHANG \\ Department of Geosciences, Florida Atlantic University, Boca Raton, Florida
}

(Manuscript received 27 July 2011, in final form 22 October 2011)

\begin{abstract}
Sea level pressure (SLP) acts, on the one hand, as a "bridge parameter" to which geophysical properties at the air-sea interface (e.g., wind stress and sea surface height) are linked, and on the other hand, as an "index parameter" by which major atmospheric oscillations, including the well-known Southern Oscillation, are defined. Using 144 yr (1854-1997) of extended reconstructed SLP data, seasonal patterns of its variability are reinvestigated in detail. New features on fundamental structure of its annual and semiannual cycles are revealed in two aspects. First, the spatiotemporal patterns of yearly and half-yearly SLPs are basically determined by a network of "amphidromes," which are surrounded by rotational variations. Fourteen cyclonic and anticyclonic annual SLP amphidromes (half each and often in pair) are found in the global ocean, while the numbers of the two types of semiannual amphidrome are 11 and 9 , respectively. The second dominant feature in SLP variability is the pattern of oscillation or seesaw for both annual and semiannual components. At least eight oscillation zones are identified for the annual cycle, which can be categorized into a boreal winter mode and an austral winter mode. As for the semiannual cycle, the seesaw pattern is geographically divided into three regimes: the North Pacific regime, the North Atlantic regime, and the Southern Ocean regime. These findings serve as a new contribution to characterizing and understanding the seasonality of the global ocean-atmosphere system.
\end{abstract}

\section{Introduction}

Sea level pressure (SLP) is the atmospheric pressure at sea level or, when measured at a given elevation on land, the station pressure reduced to sea level assuming an adiabatic lapse rate "beneath" elevated terrain. The fundamental importance of SLP in weather forecast and climate research can be understood in at least two aspects. First, it acts as a "bridge parameter" that is closely linked to a number of geophysically important variables at the air-sea interface (e.g., sea surface temperature, sea surface height, sea surface wind stress, oceanic precipitation, and sea ice extent; e.g., Fu and Pihos 1994; White and Peterson 1996; Trenberth and Caron 2000), and is therefore considered as the single most useful and

Corresponding author address: Ge Chen, College of Information Science and Engineering, Ocean University of China, 238 Songling Rd., Qingdao 266100, China.

E-mail: gechen@ouc.edu.cn valuable tool of the synoptician in diagnosing atmospheric circulation for weather and climate related studies (Simmonds, 2003). Second, it serves as an "index parameter" that defines a number of atmospheric oscillations such as the Southern Oscillation (e.g., Trenberth 1984), the North Atlantic Oscillation (e.g., Jones et al. 1997), the North Pacific Oscillation (e.g., Linkin and Nigam 2008), the Arctic Oscillation (e.g., Thompson and Wallace 1998), and the Antarctic Oscillation (e.g., Gong and Wang 1999), etc. Therefore, it is not surprising that SLP has been a constant concern of the meteorological and oceanographic community for more than a century.

With the availability of several early long-term reconstructed hemispheric datasets, the SLP climatology becomes reasonably well known (see, e.g., Figs. 7-9 in Hsu and Wallace 1976). Researches on this topic in recent decades largely focus on its variability from intraseasonal to interdecadal time scales, among which annual and semiannual cycles attract many attentions. Based on previous as well as their own works, Hsu and Wallace (1976) 
present a brief summary of progress up to mid-1970s: in the subtropical and midlatitude oceans of the Northern Hemisphere, SLPs have a maximum in summer and a minimum in late autumn. The Arctic and sub-Arctic oceans have a spring maximum in the annual cycle of SLP, a minimum in winter with a secondary maximum in November. Winter maxima are observed over the subtropical oceans of the Southern Hemisphere. There is a pronounced semiannual oscillation over the Southern Ocean with a phase reversal between $50^{\circ} \mathrm{S}$ and the Antarctic coast.

In the past 30 years, a number of studies have been carried out to describe regional characteristics of seasonal variations in SLP, such as Horel (1982) for the tropical Pacific Ocean, Simmonds and Jones (1998) for the southern extratropics, and Cullather and Lynch (2003) for the North Pole area, etc. Although significant progress has been made to characterize the annual and semiannual harmonics of SLP at both global and regional scales, a detailed view of its seasonality in terms of a joint pattern of amplitude and phase over the world's oceans is still unclear. This argument can be easily understood by a comparison with the well-defined spatiotemporal patterns of diurnal and semidiurnal tides, which also bear a periodic nature (approximately 24 and $12 \mathrm{~h}$ ) in the sense of harmonic analysis. In our study, taking advantage of the SLP data with higher resolution, longer time series, and unprecedented overall quality (see next section), new structures of global SLP seasonality are revealed, and the covariation of its amplitude and phase is investigated in detail in the context of an extended "amphidromic system."

\section{Data and method}

An extended reconstruction of monthly mean oceanic sea level pressure (ERSLP) based on the Comprehensive Ocean-Atmosphere Data Set (COADS) for the 18541997 period is used in our study (Smith and Reynolds 2004). In constructing the ERSLP dataset, the COADS data are first screened using an adaptive quality-control procedure. Land SLP data from 58 coastal and island stations are used to supplement the COADS data, which may provide better continuity near land boundaries. The SLP anomalies are analyzed monthly to a $2^{\circ} \times 2^{\circ}$ grid using statistics based on $20 \mathrm{yr}$ (1980-99) of assimilated atmospheric reanalysis. In this period the reanalysis incorporates satellite data, which makes the ERSLP more homogeneous with better coverage than over the longer reanalysis period. Validation against the post-1980 reanalysis SLP and against station data through the analysis period indicates that the new reconstruction is generally more reliable than the comparison historical analyses

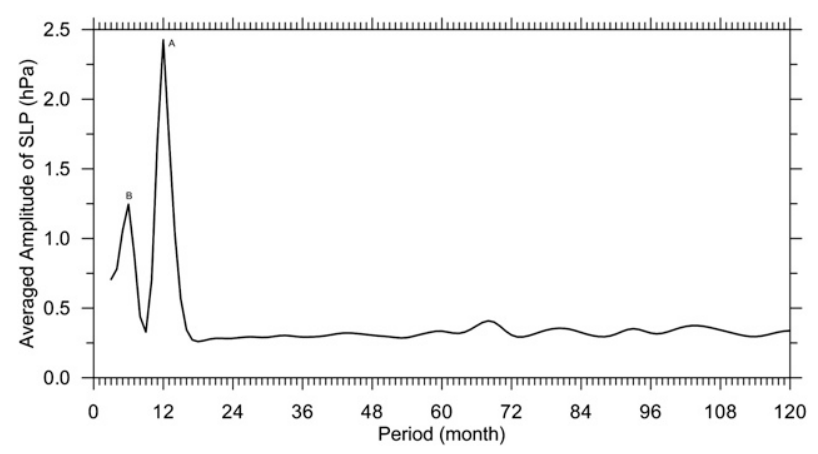

FIG. 1. Globally averaged amplitude of sea level pressure as a function of period derived from ERSLP data for 1854-1997.

(Smith and Reynolds 2004). Meanwhile, it should be noted that in the nineteenth century, the reconstruction appears to underestimate the SLP anomaly amplitudes, and error estimates for the reconstruction are largest. After 1900 the reconstruction variance is stronger, although there are periods in the first half of the twentieth century when sampling is poor and the variance decreases. Several other state-of-the-art SLP datasets are also available, such as those described in Kaplan et al. (2000), and Allan and Ansell (2006). The ERSLP dataset is chosen mainly because of its higher spatial resolution ( $2^{\circ}$ vs $4^{\circ}$ or $5^{\circ}$ longitude-latitude), which is attractive for the extraction of detailed pattern in SLP modality, though the $2^{\circ} \times 2^{\circ}$ grid is known to be inhomogeneous in terms of data quality.

Based on the data described above, the globally averaged harmonic spectrum of SLP up to $10 \mathrm{yr}$ (120 months) is shown in Fig. 1. The statistical significance of the annual and semiannual harmonics is found to be at 0.01 level. As can be seen, the annual peak labeled with $\mathrm{A}$ is nearly an order of magnitude larger than all other harmonics except for the semiannual component $\mathrm{B}$, which is approximately half of the annual component globally. These two major harmonics together are found to explain $98.23 \%$ of the total SLP variance, with annual and semiannual contributions being $90.34 \%$ and $7.89 \%$, respectively. In the remainder of this paper, we will therefore focus on the two most significant harmonics which have an absolute dominance in SLP variability.

The annual and semiannual harmonics of the seasonal cycle can be derived through Fourier analysis of the SLP time series $P(x, y, t)$ at each grid point $(x, y)$ :

$$
\begin{aligned}
& P(x, y, t)=P_{0}(x, y)+P_{1}(x, y, t)+P_{2}(x, y, t)+\varepsilon(x, y, t) \\
& P_{1}(x, y, t)=A_{1}(x, y) \times \cos \left[\left(2 \pi t / T_{1}+\varphi_{1}(x, y)\right],\right. \\
& P_{2}(x, y, t)=A_{2}(x, y) \times \cos \left[\left(2 \pi t / T_{2}+\varphi_{2}(x, y)\right],\right.
\end{aligned}
$$




$$
\varepsilon(x, y, t)=\sum_{i=3}^{6} A_{i}(x, y) \times \cos \left[2 \pi t / T_{i}+\varphi_{i}(x, y)\right],
$$

where $P_{0}(x, y)$ is the annual mean of SLP at $(x, y) ; P_{1}, P_{2}$, and $\varepsilon$ are the annual harmonic, semiannual harmonic, and sum of resolvable residual harmonics, respectively; $A_{1}$ and $A_{2}$ are the amplitudes of the yearly and halfyearly components; $\varphi_{1}$ and $\varphi_{2}$ are the phase angles that determine the times at which the maxima of the two harmonics occur, while $t$ varies from 0 to 12 months with $T_{1}=12$ months and $T_{2}=6$ months; and $i$ represents the remaining seasonal harmonics other than annual and semiannual. With the availability of a complete spatiotemporal dataset $P(x, y, t)$, the retrieved $A_{1,2}(x, y)$ and $\varphi_{1,2}(x, y)$ are supposed to carry full information of the annual and semiannual SLP variability in terms of amplitude and phase, as evidenced in Chen and Quartly (2005) and Chen and Li (2008) with sea surface temperature and sea surface height for the global ocean.

\section{Pattern of amplitude distribution}

Combining (1)-(4) with 144 yr (1854-1997) of gridded SLP data, the global distributions of annual and semiannual amplitude corresponding to peaks $\mathrm{A}$ and $\mathrm{B}$ in Fig. 1 are presented in Figs. 2 and 3, respectively. A vector representation of the phase pattern is also overlaid on Figs. 2a and 3a. As far as the annual amplitude is concerned (Fig. 2), primary maxima mainly appear in the extratropical areas of the North Pacific, Atlantic and Indian Ocean, particularly in the Kuroshio and Gulf Stream extension zones, the China Seas, as well as the Arabian Sea labeled 5, 6, 3, and 2, respectively. Secondary highs are basically observed in the Arctic Ocean (Fig. 2b), the southern Indian Ocean, the Australian coast, and the Weddell Sea along the Antarctic (Fig. 2c) labeled 1, 4, and 7-10 (Fig. 2a). In contrast, the SLP seasonality of the tropical oceans is seen to be generally indistinctive at both annual and semiannual frequencies (see also Fig. 3a). The latter, however, exhibits a somewhat different pattern at mid- to high latitudes: the largest semiannual signals are found in the Arctic Ocean (Fig. 3b), the Antarctic Ocean (Fig. 3c), and the Kuroshio extension area (Fig. 3a), labeled zones 14,10 , and 1 , respectively. It is interesting to identify three secondary SLP belts-zones for the semiannual harmonic: 1 ) a circumpolar half-yearly wave around $50^{\circ} \mathrm{S}$ in the Southern Ocean, 2) a southwest-northeast-oriented cross-Atlantic high SLP belt extending from the Gulf of Mexico to the west coast of Spain, and 3) a well-defined secondary peak in the Gulf of Alaska. Furthermore, it is worth noting that several distinct low SLP belts with regional minima are formed between major primary and secondary zones, such as those around $60^{\circ} \mathrm{S}$ in the Southern Ocean, in the midlatitude North Atlantic, and in the vicinity of the Bering Sea. In summary, the SLP variability is characterized by a systematic polar high and tropical low, with a number of midlatitude peaks-troughs (corresponding to active-inactive SLP zones) for both annual and semiannual amplitudes (note that this statement as well as the above high-low descriptions for SLP are only true in the sense of harmonic distribution rather than overall variability). Simmonds and Jones (1998) investigate the mean structure and temporal variability of the semiannual oscillation in the Southern Ocean (an earlier summary of which can be found in Van Loon 1967). They show that the half-yearly wave of SLP attains its largest values on the Antarctic periphery between $65^{\circ}$ and $75^{\circ} \mathrm{S}$, and in the midlatitude ocean basins between $30^{\circ}$ and $45^{\circ} \mathrm{S}$. The former is confirmed in our result with strongest signals in the southern Pacific sector, but the latter is seen to have a $10^{\circ}$ meridional shift to the south in our Fig. 3. Moreover, it is suspected that the cross-Atlantic semiannual oscillation belt evident in Fig. $3 \mathrm{~b}$ might be the Northern Hemisphere counterpart of the circumpolar half-year wave in the Southern Ocean (Fig. 3c), at least for the Atlantic sector. The same signature is also found in the North Pacific sector, though much weaker. These features could be a manifestation of the broader Arctic Oscillation as described by, among others, Deser (2000). She concluded that the temporal coherence between the Arctic and midlatitudes is strongest over the Atlantic sector both on intraseasonal and interannual time scales during the past 50 years, while the correlation between the Atlantic and Pacific midlatitudes is rather weak.

As far as the physical mechanism is concerned, it is understood that the SLP semiannual oscillation may result from the differing annual march of tropospheric temperature over the Southern Ocean and the Antarctic continent (Walland and Simmonds 1999). Early in the year the sun disappears and the temperatures start to drop over the Antarctic before it happens over the ocean. Similar tendencies occur at the end of the year. The rates of warming and cooling between the two annual cycles of temperature also vary because of the complex energy balance over the ocean and the landmass. Numerical model simulation experiments further demonstrate that the difference surface types and boundary conditions of the southern latitudes, the presence of the elevated Antarctic topography and extensive sea ice and its large seasonal variation, are found to have great and modest effects respectively, which give rise to twice-yearly variations in baroclinicity of the Southern Ocean (Walland and Simmonds 1998). A similar mechanism may also be 

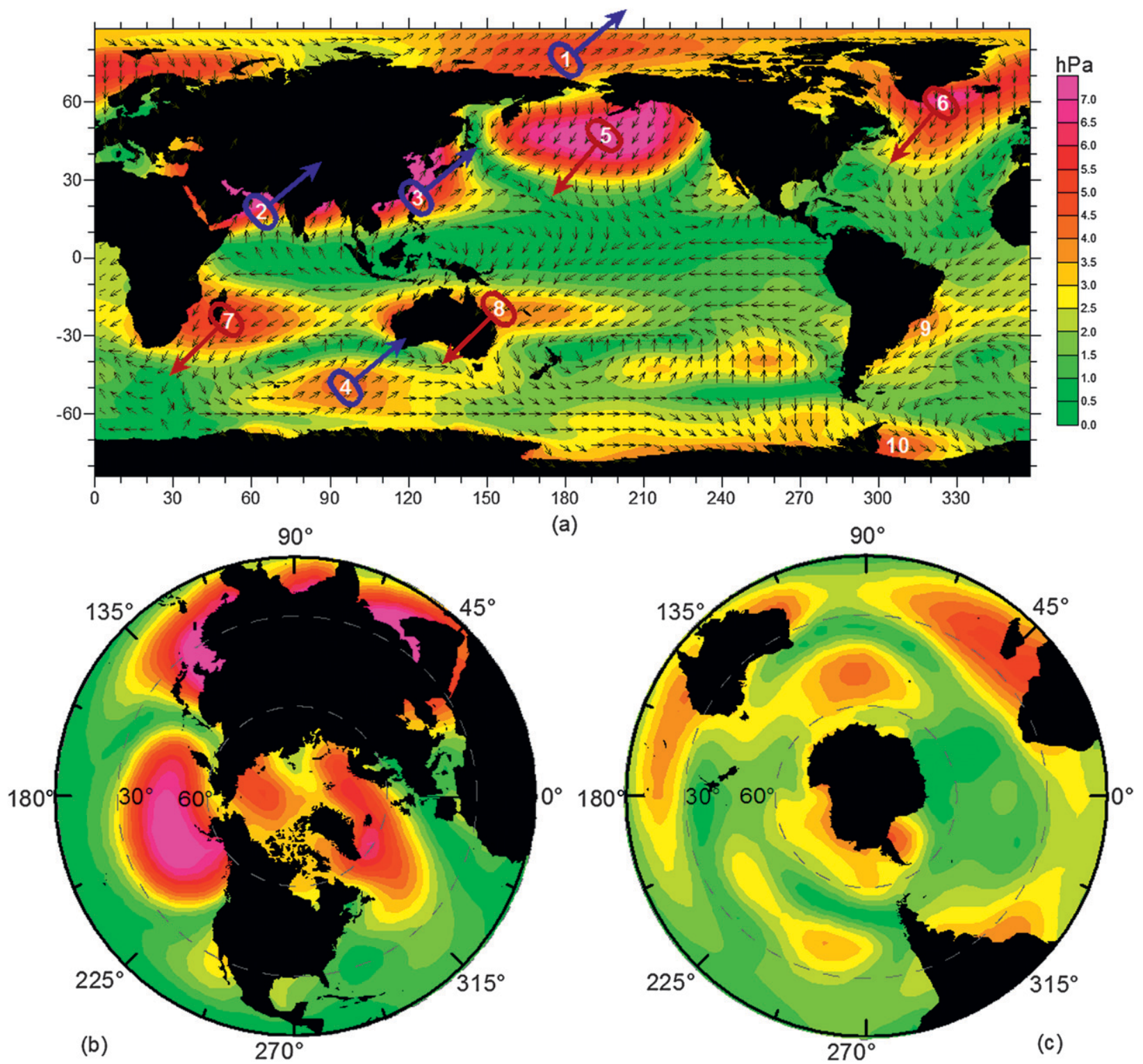

FIG. 2. (a) Geographical distribution of the annual harmonic of sea level pressure over the global oceans. Amplitude is indicated by color, and phase by orientation of the arrows. A "clock style" representation of the phase angle is adopted (i.e., "one o'clock" depicts an SLP maximum in January, while "twelve o'clock" depicts an SLP maximum in December). The large blue and brown arrows indicate SLP peaks occurring during the first and second half of the respective period, with number labels corresponding to identified SLP active zones. The polar stereographic versions of (a), but for the (b) Northern and (c) Southern Hemispheres.

applied to the Northern Hemisphere in the context of the Arctic Ocean and the surrounding continents but in a reverse land-ocean manner, at least for the Atlantic and Pacific sectors (Fig. 3b).

\section{Pattern of phase distribution}

Next, we examine the annual and semiannual phase patterns of SLP variation as shown in Figs. 4 and 5, where the color bar indicates the calendar month in which the annual-semiannual amplitude peaks. A striking fundamental feature shared by Figs. 4 and 5 is that the phase distribution is dominated by a dozen of complex yet clearly defined amphidromic systems as commonly seen in cophase line maps of ocean tides. The scientific term "amphidrome" is usually associated with a rotary tide in oceanography. It normally refers to a "no tide" point in the sea where the amplitude is zero and the phase is undetermined for a given tidal component. In fact, amphidromes are nodes of its cophase lines, and the high tide 

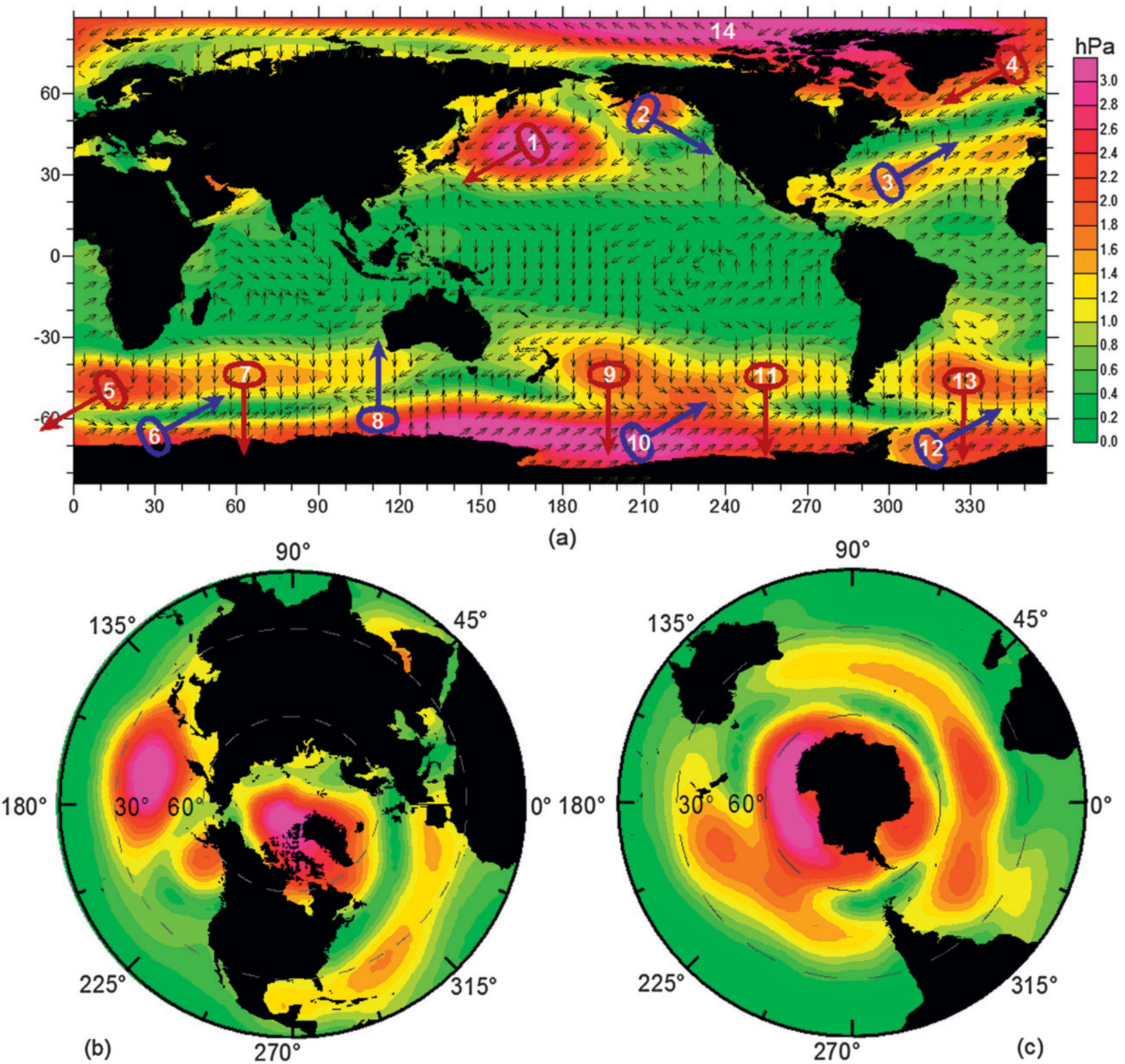

(a)

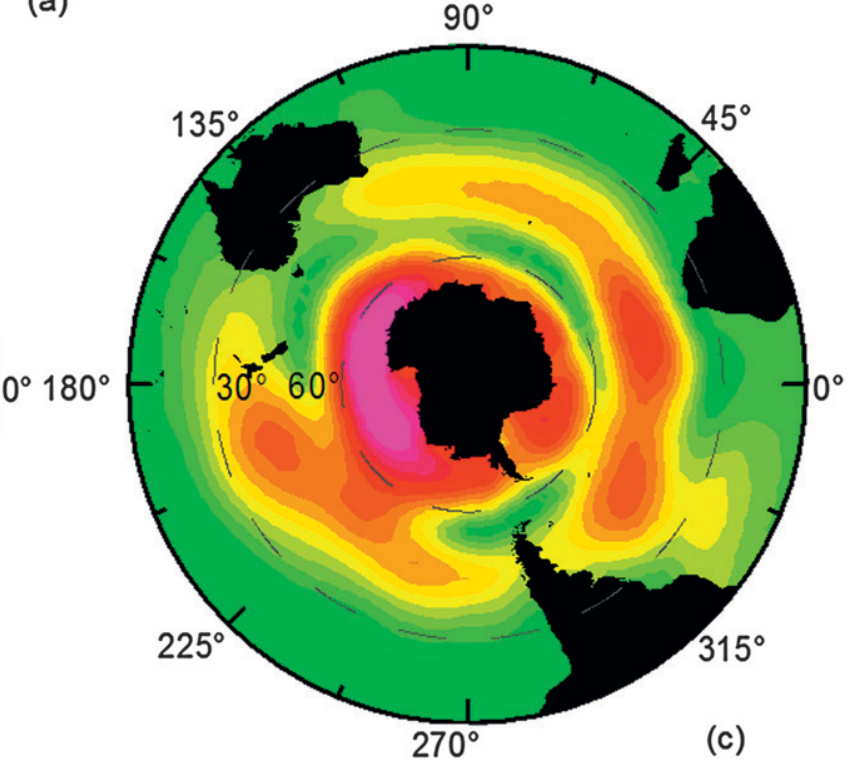

FIG. 3. As in Fig. 2, but for the semiannual harmonic. "Two o'clock" depicts an SLP maximum in January and July, while "twelve o'clock" depicts an SLP maximum in June and December.

crest occurs at the same time along each cophase line. The distance between low and high water levels, or the tidal range, for a rotary tide is shown by a series of lines decreasing in value as they approach the amphidromic point. Thus, amphidromes are naturally linked with amplitude and phase, which, in combination with periodicity, constitute the three basic parameters to characterize the so-called harmonic signal. This concept can also be extended to nontidal oceanographic and atmospheric variables with a periodic nature (Chen and Quartly 2005; Zhang et al. 2008). Amphidromes are therefore considered as critical nodal points that define the fundamental pattern of the spatiotemporal variation of a geophysical property. Two types of amphidrome are found to scatter over the world's oceans: cyclonic and anticyclonic, around which the SLP harmonic waves rotate anticlockwise-clockwise with time on a periodic basis. Each type has seven realizations for the annual component (Fig. 4a). For the semiannual component, however, the numbers of identified cyclonic and anticyclonic amphidromes are 11 and 9, respectively. Many of these amphidromes appear in pair, since they usually share several cophase lines in common. But there is no obvious geographical correlation between annual and semiannual 

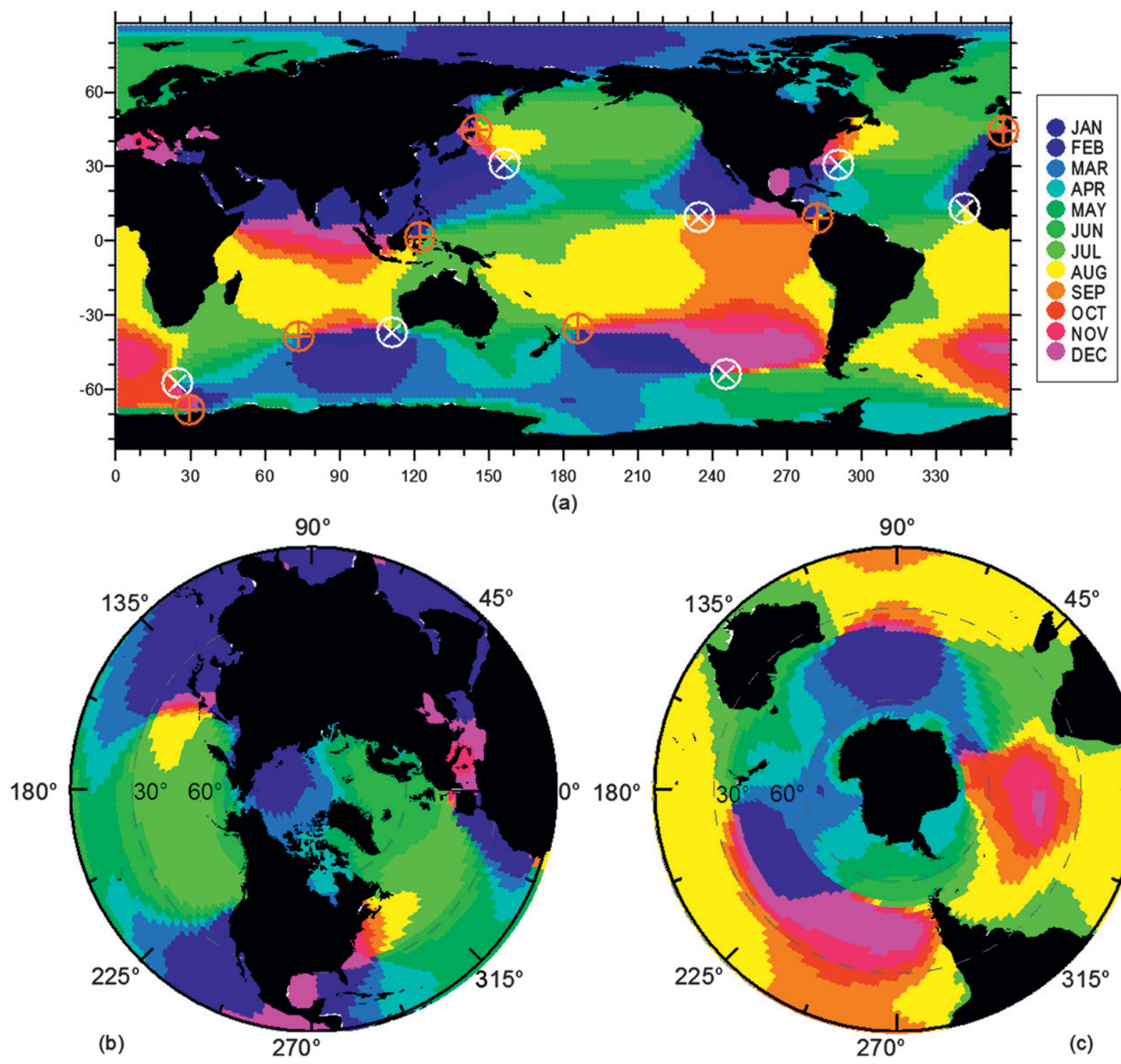

(a)

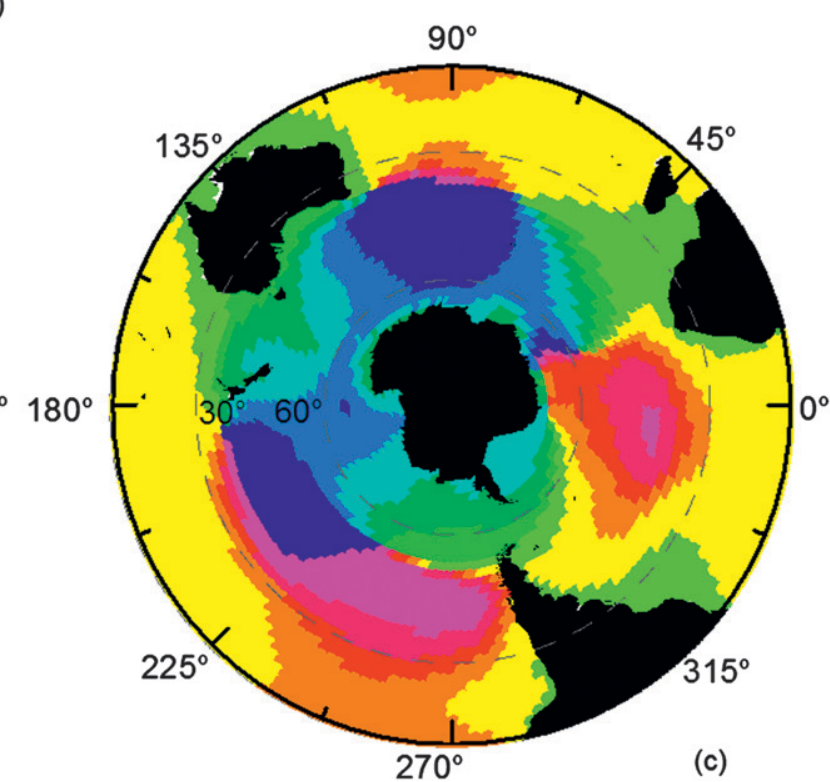

FIG. 4. (a) Geographical distribution of the annual phase of sea level pressure variations over the global oceans. The color bar indicates the calendar month in which the annual SLPs peak. The symbols " $\oplus$ " and " $\otimes$ " indicate cyclonic and anticyclonic amphidromes, respectively. The polar stereographic versions of (a), but for the (b) Northern and (c) Southern Hemispheres.

amphidromes. The existence of these systematic amphidromes determines that the phase pattern of SLP is basically rotational rather than zonal. Therefore, the latitudinally banded description by Hsu and Wallace (1976), among others, as summarized in the introduction reveals only an incomplete and perhaps distorted picture of the global SLP phase variation.

Similar "misleading" characterizations are also found in previous regional studies on SLP variability. In his research on the annual cycle of the tropical Pacific atmosphere and ocean, Horel (1982) finds that, near the equator, the highest pressures occur during July and
August, while between $10^{\circ}$ and $20^{\circ} \mathrm{N}$ in the central ocean they are largest during March and April. In fact, a September maximum appears in eastern equatorial Pacific, while a varying August-December peak can be found in the equatorial Indian Ocean due to the existence of a clockwise annual amphidrome near the Celebes Sea (see Fig. 4a). Using reanalysis and in situ data, Cullather and Lynch (2003) present a generalized depiction of the annual cycle of pressure fields over the Arctic. They show that above the Canada Basin-Laptev Sea side of the Arctic, the annual cycle of surface pressure is dominated by the first harmonic, whose amplitude is about $5 \mathrm{hPa}$ 


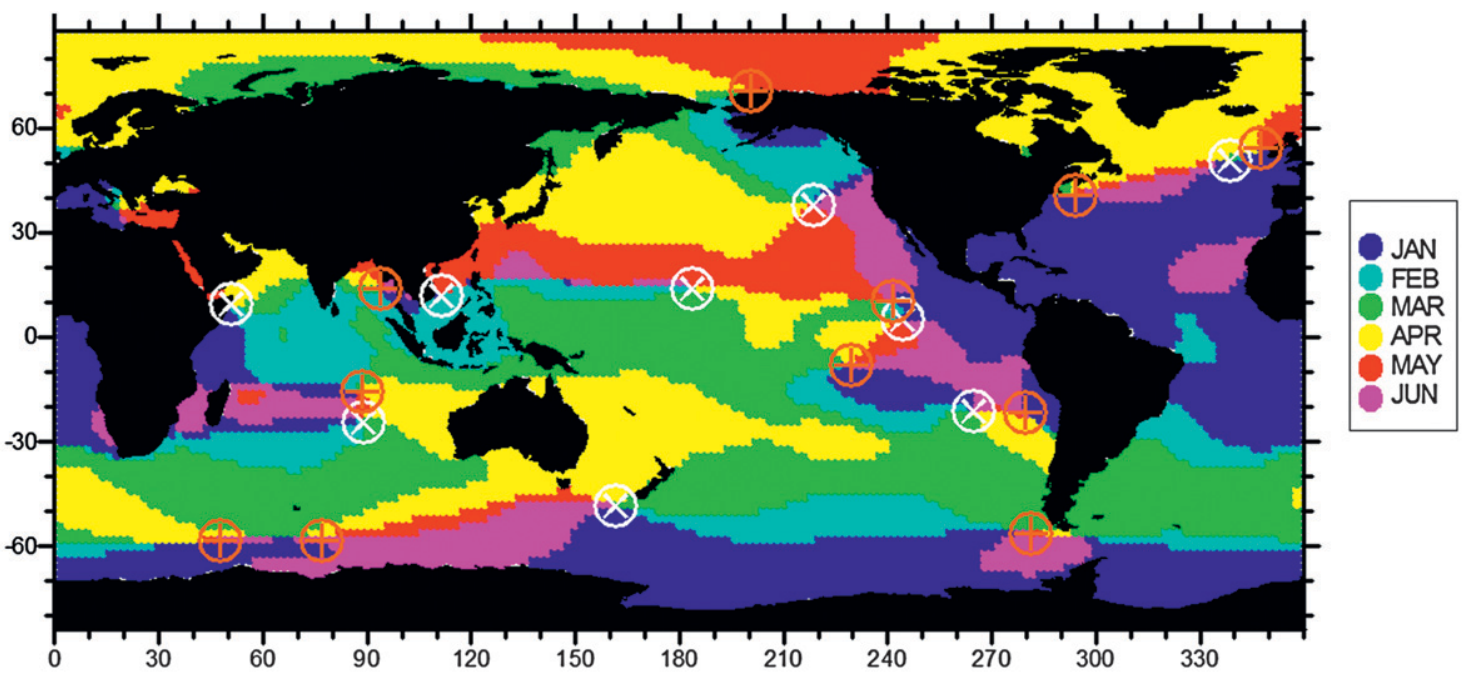

(a)
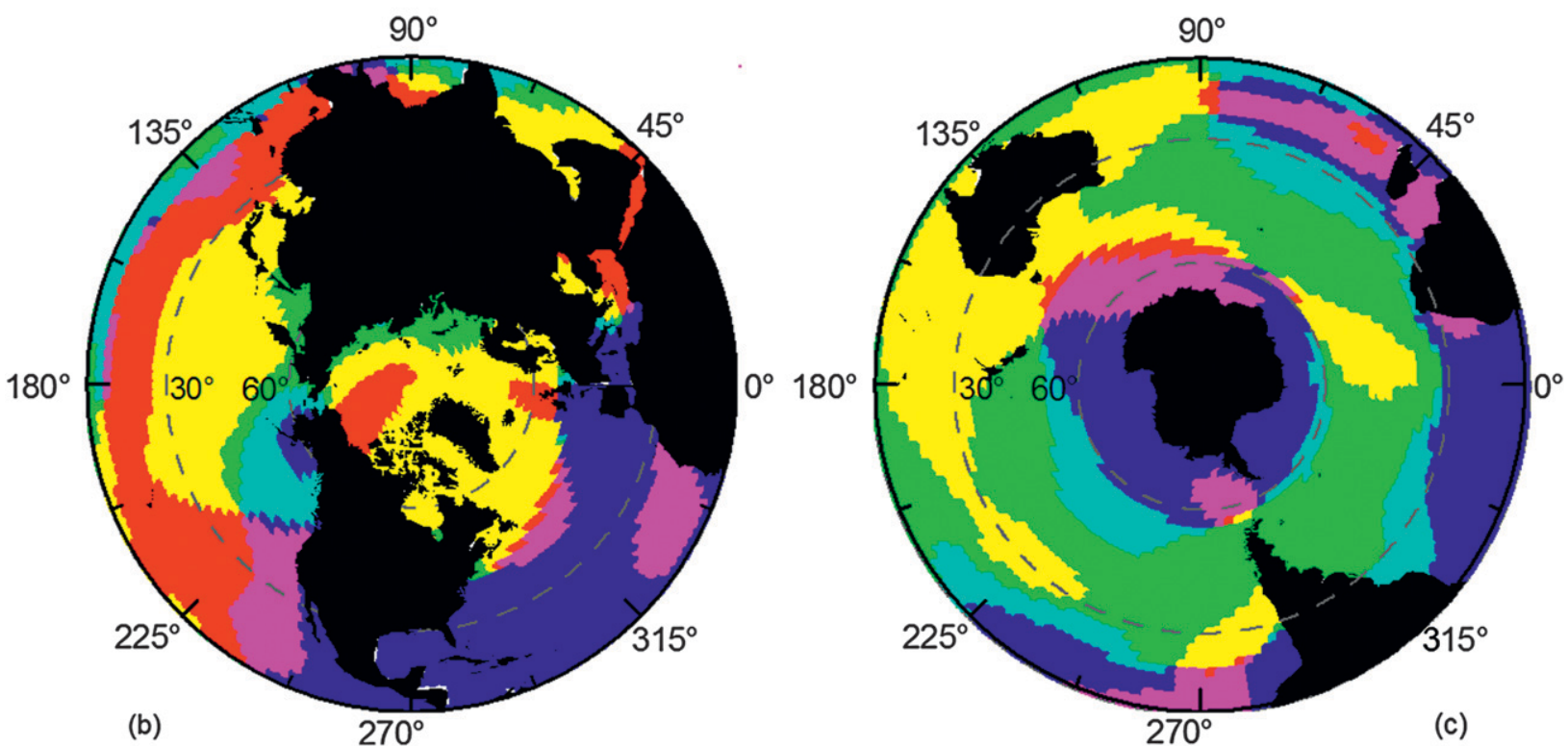

FIG. 5. As in Fig. 4, but for the semiannual phase.

with maximum pressure occurring in March. Along the periphery of northern Greenland and extending to the North Pole, a weak semiannual cycle is observed in surface pressure with maxima in May and November. Our result suggests that the annual peak of SLP in the Arctic Ocean occurs during the first half of the year: progressing from February in the Laptev Sea-East Siberian Sea, both eastward and westward, to June in the Greenland Sea (Fig. 4b). In contrast, the first peak of the semiannual pressure wave arrives in April for the majority of the Arctic Ocean, except for the Barents Sea-East Siberian Sea areas that advance to March, and for the vast waters extending from the Beaufort Sea to the North Pole, which delay until May (Fig. 5b).

\section{A joint view of amplitude and phase: Seasonal seesaws}

A joint view of amplitude and phase allows us to identify oscillation patterns in seasonal SLP variations. It is understood that major oscillation zones should be associated with amplitude highs labeled 1-10 in Fig. 2a and 1-14 in Fig. 3a. As far as the annual cycle is concerned, two preferable timings are apparent for SLP to peak: January-February and July-August, as indicated with large blue and brown arrows in Fig. 2a. This leads to a phase reversal, or oscillation, for major SLP active zones. An alternating high-low pattern can therefore be expected around $75^{\circ} \mathrm{N}$ (zone 1), $50^{\circ} \mathrm{N}$ (zones 5 and 6), 
$20^{\circ} \mathrm{N}$ (zones 2 and 3 ), $20^{\circ} \mathrm{S}$ (zones 7 and 8), and $50^{\circ} \mathrm{S}$ (zone 4) extending from north to south. As a result, a number of regional oscillations are geographically formed, such as zones 1 versus 5, 3 versus 5, 4 versus 7, and so on.

As for the semiannual cycle, there are basically three oscillation regimes: the North Pacific regime, the North Atlantic regime, and the Southern Ocean regime (Fig. 3a). In the North Pacific, the primary SLP maximum (zone 1) is delayed by two months with respect to the secondary maximum (zone 2). In contrast, a sharp phase opposition corresponding to a strong seesaw of semiannual SLP is evident in northern Atlantic (zones 3 and 4) with a transitional low-amplitude belt in between. These results provide an evident support to the argument that the "annular" character of the Arctic Oscillation is more a reflection of the dominance of its Arctic center of action than any coordinated behavior of the Atlantic and Pacific centers of action in the SLP field (Deser 2000). The third regime exists across the entire Southern Ocean with zones 5, 7, 9, 11, and 13 peaking around March and September, while zones $6,8,10$, and 12 peak around January and July, between which the Antarctic Circumpolar Current flows along an inactive semiannual belt within $50^{\circ}-60^{\circ} \mathrm{S}$ where a number of SLP amphidromes reside (see also Fig. 5a). Our result is consistent with the report by Simmonds and Jones (1998) that a general minimum lies between $55^{\circ}$ and $60^{\circ} \mathrm{S}$, marking the region where the phase of the harmonic shifts from an equinox maximum in the midlatitudes to an equinox minimum at high latitudes.

\section{Conclusions}

In this study, the annual and semiannual cycles of global SLP are revisited using the gridded ERSLP data with an emphasis on coupled amplitude-phase variability derived from harmonic analysis. New aspects of their fundamental patterns in the context of "amphidrome" and "oscillation" are revealed.

For the majority of the world's oceans, the SLP distribution is dominated by a network of amphidromes. The SLP variation is largely rotational rather than zonal or regional as previously described. A realistic understanding of its detailed spatiotemporal pattern can be effectively obtained by combining amplitude and phase distributions in an amphidromic context. Generally, a highamplitude area corresponds to a slowly varying phase pattern, and vice versa (i.e., a zero or small-amplitude area corresponds to a large phase gradient, exhibiting a typical amphidromic scenario). Specifically, 14 cyclonic and anticyclonic annual SLP amphidromes (half each and often in pair) are found in the global ocean (Fig. 4a), and the numbers of the two types of semiannual amphidrome are 11 and 9, respectively (Fig. 5a). These include welldefined major amphidromes and distorted minor amphidromes. Such an important signature is obviously overlooked in many of the previous studies with coarsely distributed data.

Another striking feature in SLP variability is the pattern of oscillation or seesaw, which is significant for both annual and semiannual components. An oscillation is formed between two neighboring areas with high SLP amplitudes and a phase opposition. At least eight oscillation zones can be identified for the annual cycle (Fig. 2), which can be categorized into a boreal winter (JanuaryFebruary) mode (zones 1-4) and an austral winter (JulyAugust) mode (zones 5-8). Zones in one mode are usually $180^{\circ}$ out of phase with those in the other. As a result, an oscillation or a teleconnection exists between any of the large blue and brown arrow zones in Figs. 2a and 3a. As for the semiannual cycle, the seesaw pattern is geographically divided into three regimes: the North Pacific regime, the North Atlantic regime, and the Southern Ocean regime (Fig. 3a). The North Atlantic regime is characterized by a typical phase reversal between zones 3 and 4, while the other two regimes are seen to have a general phase opposition with a 1-2 months advance-delay. A belt of amplitude low is observed between the opposite phase zones within each regime.

Finally, it should be pointed out that the analysis in this paper is based on the mean annual SLP cycle averaged over 144 years. The identified structures are subject to certain variabilities at interannual, decadal, and centennial time scales, especially for the semiannual signals as evidenced both in observations and in models (e.g., Meehl et al. 1998; Simmonds and Walland 1998). These large-scale amphidromic and oscillation patterns as well as their variations are believed to play a key role in defining and modifying the seasonality of the global oceanatmosphere system.

Acknowledgments. This research was jointly supported by the Global Change Research Program of China under Project 2012CB955603, the Natural Science Foundation of China under Projects 40730530 and 41076115, the National Basic Research Program of China under Project 2009CB723903, and the National High-Tech Research and Development Program of China under Project 2008AA121701. The authors wish to thank Haoyu Jiang for his assistance in producing some of the color plots.

\section{REFERENCES}

Allan, R., and T. Ansell, 2006: A new globally complete monthly historical gridded mean sea level pressure dataset (HadSLP2): 1850-2004. J. Climate, 19, 5816-5842. 
Chen, G., and G. D. Quartly, 2005: Annual amphidrome: A common feature in the ocean? IEEE Geosci. Remote Sens. Lett., 2, $423-427$.

- and H. Li, 2008: Fine pattern of natural modes in sea surface temperature variability: 1985-2003. J. Phys. Oceanogr., 38, 314-336.

Cullather, R. I., and A. H. Lynch, 2003: The annual cycle and interannual variability of atmospheric pressure in the vicinity of the North Pole. Int. J. Climatol., 23, 1161-1183.

Deser, C., 2000: On the teleconnectivity of the "Arctic Oscillation." Geophys. Res. Lett., 27, 779-782.

Fu, L.-L., and G. Pihos, 1994: Determining the response of sea level to atmospheric pressure forcing using TOPEX/POSEIDON data. J. Geophys. Res., 99, 24 633-24 642.

Gong, D., and S. Wang, 1999: Definition of the Antarctic oscillation index. Geophys. Res. Lett., 26, 459-462.

Horel, J. D., 1982: On the annual cycle of the tropical Pacific atmosphere and ocean. Mon. Wea. Rev., 110, 1863-1878.

Hsu, C.-P., and J. M. Wallace, 1976: The global distribution of the annual and semiannual cycles in sea level pressure. Mon. Wea. Rev., 104, 1597-1601.

Jones, P. D., T. Jonsson, and D. Wheeler, 1997: Extension to the North Atlantic Oscillation using early instrumental pressure observations from Gibraltar and south-west Iceland. Int. J. Climatol., 17, 1433-1450.

Kaplan, A., Y. Kushnir, and M. A. Cane, 2000: Reduced space optimal interpolation of historical marine sea level pressure: 1854-1992. J. Climate, 13, 2987-3002.

Linkin, M. E., and S. Nigam, 2008: The North Pacific OscillationWest Pacific teleconnection pattern: Mature-phase structure and winter impacts. J. Climate, 21, 1979-1996.

Meehl, G. A., J. W. Hurrell, and H. Van Loon, 1998: A modulation of the mechanism of the semiannual oscillation in the Southern Hemisphere. Tellus, 50A, 442-450.
Simmonds, I., 2003: Modes of atmospheric variability over the Southern Ocean. J. Geophys. Res., 108, 8078, doi:10.1029/2000JC000542.

— , and D. A. Jones, 1998: The mean structure and temporal variability of the semiannual oscillation in the southern extratropics. Int. J. Climatol., 18, 473-504.

_ , and D. J. Walland, 1998: Decadal and centennial variability of the southern semiannual oscillation simulated in the GFDL coupled GCM. Climate Dyn., 14, 45-53.

Smith, T. M., and R. W. Reynolds, 2004: Reconstruction of monthly mean oceanic sea level pressure based on COADS and station data (1854-1997). J. Atmos. Oceanic Technol., 21, 1272-1282.

Thompson, D. W. J., and J. M. Wallace, 1998: The Arctic oscillation signature in wintertime geopotential height and temperature fields. Geophys. Res. Lett., 25, 1297-1300.

Trenberth, K. E., 1984: Signal versus noise in the Southern Oscillation. Mon. Wea. Rev., 112, 326-332.

, and J. M. Caron, 2000: The Southern Oscillation revisited: Sea level pressures, surface temperatures, and precipitation. J. Climate, 13, 4358-4365.

Van Loon, H., 1967: The half-yearly oscillations in the middle and high southern latitudes and the coreless winter. J. Atmos. Sci., 24, 472-486.

Walland, D., and I. Simmonds, 1998: Sensitivity of the Southern Hemisphere semiannual oscillation in surface pressure to changes in surface boundary conditions. Tellus, 50A, 424-441.

$\longrightarrow$, and $\longrightarrow$ 1999: Baroclinicity, meridional temperature gradients, and the southern semiannual oscillation. J. Climate, 12, 3376-3382.

White, B. W., and R. G. Peterson, 1996: An Antarctic circumpolar wave in surface pressure, wind, temperature and sea ice extent. Nature, 380, 699-702.

Zhang, C., G. Chen, and F. Qiu, 2008: Annual amphidromes observed in the atmosphere with remote sensing data. J. Geophys. Res., 113, D16112, doi:10.1029/2008JD009864. 